\title{
Selektion von Geschäftsprozessen zur Anwendung von Robotic Process Automation am Beispiel einer Versicherung
}

\author{
Mathias Eggert $(\mathbb{D} \cdot$ Tobias Moulen
}

Eingegangen: 26. März 2020 / Angenommen: 30. September 2020 / Online publiziert: 19. Oktober 2020 (C) Der/die Autor(en) 2020

Zusammenfassung Häufig bremsen geringe IT-Ressourcen, fehlende Softwareschnittstellen oder eine veraltete und komplex gewachsene Systemlandschaft die Automatisierung von Geschäftsprozessen. Robotic Process Automation (RPA) ist eine vielversprechende Methode, um Geschäftsprozesse oberflächenbasiert und ohne größere Systemeingriffe zu automatisieren und Medienbrüche abzubauen. Die Auswahl der passenden Prozesse ist dabei für den Erfolg von RPA-Projekten entscheidend. Der vorliegende Beitrag liefert dafür Selektionskriterien, die aus einer qualitativen Inhaltanalyse von elf Interviews mit RPA-Experten aus dem Versicherungsumfeld resultieren. Das Ergebnis umfasst eine gewichtetet Liste von sieben Dimensionen und 51 Prozesskriterien, welche die Automatisierung mit Softwarerobotern begünstigen bzw. deren Nichterfüllung eine Umsetzung erschweren oder sogar verhindern. Die drei wichtigsten Kriterien zur Auswahl von Geschäftsprozessen für die Automatisierung mittels RPA umfassen die Entlastung der an dem Prozess mitwirkenden Mitarbeiter (Arbeitnehmerüberlastung), die Ausführbarkeit des Prozesses mittels Regeln (Regelbasierte Prozessteuerung) sowie ein positiver KostenNutzen-Vergleich. Praktiker können diese Kriterien verwenden, um eine systematische Auswahl von RPA-relevanten Prozessen vorzunehmen. Aus wissenschaftlicher Perspektive stellen die Ergebnisse eine Grundlage zur Erklärung des Erfolgs und Misserfolgs von RPA-Projekten dar.

Schlüsselwörter Robotic Process Automation - RPA · Prozessautomatisierung · Prozessverbesserung · Selektionskriterien · Prozessauswahl

\footnotetext{
M. Eggert $(\bowtie)$

Fachhochschule Aachen, Eupener Str. 70, 52066 Aachen, Deutschland

E-Mail: eggert@fh-aachen.de

T. Moulen

Gothaer Systems GmbH, Gothaer Allee 1, 50969 Köln, Deutschland

E-Mail: tobias.moulen@gothaer.de
} 


\title{
Selection of business processes for the application of Robotic Process Automation using the example of an insurance company
}

\begin{abstract}
Often, low IT resources, missing APIs or an outdated and complex system landscape slow down the automation of business processes. Robotic Process Automation (RPA) is a promising method to automate business processes in a UIbased manner and without major system changes as well as to reduce media breaks. The selection of suitable processes is crucial for the success of RPA projects. This article provides process selection criteria, which result from a qualitative content analysis of eleven interviews with RPA experts from the insurance sector. The result is a weighted list of seven dimensions and 51 process criteria that favor automation with software robots or whose non-fulfillment makes implementation difficult or even impossible. The three most important criteria for selecting business processes for automation using RPA include the work load reducton of the employees involved in the process (employee overload), the executability of the process using rules (rule-based process control) and a positive cost-benefit comparison. Practitioners can use these criteria to make a systematic selection of RPA-relevant processes. From a scientific perspective, the results provide a basis for explaining the success and failure of RPA projects.
\end{abstract}

Keywords Robotic Process Automation · RPA · Process automation · Process improvement $\cdot$ Selction criteria $\cdot$ Process selection

\section{Einleitung und Motivation}

Häufig bremsen geringe IT-Ressourcen, fehlende Softwareschnittstellen oder eine veraltete und komplexe Systemlandschaft die Automatisierung der Ausführung von Geschäftsprozessen (Syed et al. 2020; Beetz und Riedl 2019). Robotic Process Automation (RPA) ist eine vielversprechende Methode, um Geschäftsprozesse oberflächenbasiert zu automatisieren und Medienbrüche abzubauen. Im Gegensatz zu traditionellen Formen der systembasierten Prozessverbesserung benötigen Software-Roboter keine invasiven Eingriffe in die Systemlandschaft. Benutzerfreundliche Tools, wie Rekorder und „Drag-and-Drop“-Baukästen versetzen auch Fachexperten ohne tiefgreifende, technische Kompetenzen in die Lage, Roboter zu trainieren und ihre eigenen Prozesse zu automatisieren.

Die Anzahl und Komplexität der existierenden Geschäftsprozesse erfordert eine systematische Vorgehensweise, um unter den gegebenen Rahmenbedingungen die Prozesse zu identifizieren, für die RPA gewinnbringend eingesetzt werden kann. Bei der Auswahl der richtigen Prozesse spielen die Eigenschaften der Geschäftsprozesse eine besondere Rolle (van der Aalst et al. 2018). Zu relevanten Eigenschaften wurden bereits Untersuchungen durchgeführt und erste Vorschläge für Auswahlkriterien entwickelt (z. B. Syed et al. 2020; Beetz und Riedl 2019; Lacity und Willcocks 2016; Fung 2014). Qualitativ empirische Untersuchungen sind bislang die Ausnahme.

Der vorliegende Beitrag adressiert die praxisnahe Erhebung von Selektionskriterien und beantwortet Interview-basiert die Frage, welche Eigenschaften ein Ge- 
schäftsprozess besitzen sollte, um durch RPA gewinnbringend unterstützt zu werden. Im Ergebnis liefert dieser Beitrag eine gewichtete Liste von Prozesskriterien, welche die Automatisierung mit Softwarerobotern begünstigen bzw. deren Nichterfüllung eine Umsetzung erschweren oder sogar verhindern kann. Der Beitrag endet mit Vorschlägen für weitere auf den Ergebnissen aufbauenden Forschungsarbeiten.

\section{Robotic Process Automation und Selektionskriterien für Prozesse}

Robotic Process Automation (RPA) beschreibt eine aufstrebende Form der Geschäftsprozessautomatisierung (Aguirre und Rodriguez 2017), die mit Hilfe virtueller Roboter bestehende Anwendungssysteme eines Unternehmens bedient (Sutherland 2013). Entgegen der Vorstellung physischer Roboter, die in einem Büro agieren, handelt es sich um Software, welche die Bezeichnung „Roboter“ auf Grund der Imitierung menschlicher Interaktionen auf Benutzeroberflächen erhält (Lacity und Willcocks 2016).

Anders als herkömmliche Werkzeuge der Prozessautomatisierung stellt RPA eine non-invasive Technologie dar, die keine Veränderung der bestehenden IT-Infrastruktur eines Unternehmens erfordert (Beetz und Riedl 2019; Willcocks et al. 2015). Vielmehr agiert die Technologie in einer ,outside-in“-Manier auf der vorhandenen Systemlandschaft, indem lediglich auf bestehende Systemschnittstellen, wie UserInterfaces zugegriffen wird (Sutherland 2013; van der Aalst et al. 2018).

Hierzu kann ein Roboter von einem fachlichen Experten ohne IT-Kenntnisse ,trainiert" bzw. konfiguriert werden. Dabei sind keine oder nur begrenzte Programmierkenntnisse notwendig (Willcocks et al. 2015). Denn im Gegensatz zu den meisten BPMN-Softwarepaketen werden keine Programmierfähigkeiten für die Konfiguration der Roboter benötigt (Aguirre und Rodriguez 2017).

Zur Selektion von geeigneten Prozessen existieren bereits erste Arbeiten (z.B. Syed et al. 2020; Beetz und Riedl 2019; Lacity und Willcocks 2016; Fung 2014). Insbesondere die Anzahl der Prozessinstanzen und involvierter Systeme sowie eine regelbasierte Prozesssteuerung werden dabei immer wieder genannt. Existierenden Beiträgen ist gemein, dass sie hauptsächlich argumentativ und erfahrungsbasiert Selektionskriterien erheben. Experteninterviews wie bei Beetz und Riedl (2019) und Fung (2014) wurden bisher selten durchgeführt, was den vorliegenden Beitrag motiviert.

\section{Methodisches Vorgehen}

Um praxisrelevante Kriterien für die Auswahl von Geschäftsprozessen für RPA zu erhalten, wurde eine Qualitative Inhaltsanalyse mit induktiver Kategorienbildung nach Mayring (2010) durchgeführt. Um eine hohe Ergebnisqualität zu erzielen, wurden ausschließlich Personen befragt, die Erfahrung in mindestens einer der von Smeets et al. (2019) definierten Rollen einer RPA-Unit gesammelt haben (RPAManager, RPA-Business-Analyst, RPA-Entwickler und RPA-Solution-Architect). 
Zur Durchführung des Interviews wurde ein Leitfaden entwickelt, dessen Aufbau in fünf Phasen unterteilt ist. Nach einer kurzen Vorstellung, einer thematischen Einleitung und der Warm-up-Phase folgen im Hauptteil konkrete Fragen zum Nutzen von RPA für das Unternehmen, zum Vergleich geeigneter Prozesse bzw. ihrer Eigenschaften und zu den wichtigsten Selektions- und Ausschlusskriterien aus Sicht des Experten. Zudem sollen wichtige Informationsquellen und Einflussfaktoren ermittelt sowie ein Vergleich zu anderen Automatisierungskonzepten oder der manuellen Bearbeitung aufgestellt werden. In der Cool-off-Phase wird dem Interviewpartner Raum für ergänzende Erläuterungen gegeben. Das Interview endet mit einer Verabschiedung sowie Hinweisen zur anonymen Auswertung und den nächsten Schritten. Alle Interviews werden mit Hilfe eines digitalen Aufnahmegerätes aufgezeichnet und transkribiert.

Die Analyse umfasst die regelbasierte Zuordnung des Textmaterials zu induktiv abgeleiteten Prozesskriterien. In Anlehnung an Vogelsang et al. (2013) bilden die Entwicklung der Kriterien, die Bestimmung ihrer eindeutigen Definitionen und das Kodieren des Materials einen iterativen Prozess, der auch in der durchgeführten Analyse angewendet wird. Um die Relevanz der Aussagen zu bewerten, wird die Häufigkeit der Kategorie-Nennungen erhoben und verglichen. Dazu werden die kodierten Aussagen durch das adaptierte Vorgehen von Vogelsang et al. (2013),

Tab. 1 Demografische Daten der Interviewteilnehmer

\begin{tabular}{|c|c|c|c|c|c|c|c|}
\hline ID & $\begin{array}{l}\text { Ge- } \\
\text { schlecht }\end{array}$ & Qualifikation & Alter $^{\mathrm{a}}$ & $\begin{array}{l}\text { Berufs- } \\
\text { erfahrung }\end{array}$ & $\begin{array}{l}\text { RPA } \\
\text { Erfahrung }^{\mathrm{a}}\end{array}$ & RPA Rolle & Bereich \\
\hline $\mathrm{A}$ & M & $\begin{array}{l}\text { Master Mathematik } \\
\text { (Non-IT) }\end{array}$ & 30 & 5 & 2 & Entwickler & TG A \\
\hline B & M & Bachelor WI (IT) & 26 & 5 & 2 & Entwickler & TG A \\
\hline $\mathrm{C}$ & M & $\begin{array}{l}\text { Master BWL } \\
\text { (Non-IT) }\end{array}$ & 39 & 11 & 2 & Manager & DG \\
\hline $\mathrm{D}$ & M & $\begin{array}{l}\text { Promotion Informatik } \\
\text { (Non-IT) }\end{array}$ & 48 & 20 & 2 & Manager & DG \\
\hline $\mathrm{E}$ & M & Dipl. WI (IT) & 33 & 13 & 1 & $\begin{array}{l}\text { Solution- } \\
\text { Architect }\end{array}$ & TG C \\
\hline $\mathrm{F}$ & M & Bachelor WI (IT) & 23 & 6 & 3 & $\begin{array}{l}\text { Business- } \\
\text { Analyst }\end{array}$ & DG \\
\hline G & $\mathrm{W}$ & $\begin{array}{l}\text { Master Risk and In- } \\
\text { surance Management } \\
\text { (Non-IT) }\end{array}$ & 27 & 5 & 1 & $\begin{array}{l}\text { Business- } \\
\text { Analyst }\end{array}$ & TG B \\
\hline $\mathrm{H}$ & M & $\begin{array}{l}\text { Betriebswirt \& Dipl. } \\
\text { Informatik (IT) }\end{array}$ & 53 & 25 & 2 & $\begin{array}{l}\text { Business- } \\
\text { Analyst }\end{array}$ & TG A \\
\hline $\mathrm{I}$ & M & Volljurist (Non-IT) & 54 & 25 & 1 & $\begin{array}{l}\text { Business- } \\
\text { Analyst }\end{array}$ & $\mathrm{ED}$ \\
\hline $\mathrm{J}$ & M & Dipl. Physik (Non-IT) & 50 & 25 & 3 & $\begin{array}{l}\text { Business- } \\
\text { Analyst }\end{array}$ & ED \\
\hline $\mathrm{K}$ & M & $\begin{array}{l}\text { Datenbankfachmann } \\
\text { (IT) }\end{array}$ & 52 & 28 & 2 & $\begin{array}{l}\text { Business- } \\
\text { Analyst }\end{array}$ & $\mathrm{ED}$ \\
\hline
\end{tabular}

TG Tochtergesellschaft, DG Dachgesellschaft/Holding, ED Externer Dienstleister, WI Wirtschaftsinformatik

aAngabe in Jahren 
Tab. 2 Selektionskriterien für RPA-geeignete Prozesse

\begin{tabular}{|c|c|c|c|}
\hline Dimension und Selektionskriterium & $\begin{array}{l}\text { Interview- } \\
\text { Frequenz }\end{array}$ & $\begin{array}{l}\text { Aussagen- } \\
\text { Frequenz }\end{array}$ & Relevanz \\
\hline \multicolumn{4}{|l|}{ Prozess-Stakeholder } \\
\hline Arbeitnehmerüberlastung (B) & 10 & 34 & 33 \\
\hline Verfügbare Fachkräfte (E) & 7 & 12 & 18 \\
\hline Steigerung der Kundenzufriedenheit (B) & 9 & 15 & 16 \\
\hline Vorschlag eines Fachbereichs (E) & 6 & 14 & 14 \\
\hline Akzeptanz der Fachkräfte (E) & 6 & 9 & 12 \\
\hline Vorhandene Entscheidungsträger (E) & 4 & 6 & 5 \\
\hline Einbeziehung der Betriebsräte (E) & 2 & 4 & 5 \\
\hline Unternehmenspolitische Relevanz (B/E) & 3 & 4 & 4 \\
\hline \multicolumn{4}{|l|}{ Qualitative Prozesseigenschaften } \\
\hline Regelbasierte Prozessteuerung (E) & 11 & 22 & 26 \\
\hline Niedriger Komplexitätsgrad (E) & 11 & 28 & 20 \\
\hline Zugriff auf strukturierte Daten (E) & 9 & 15 & 17 \\
\hline Vorangegangene Prozessoptimierung (E) & 8 & 15 & 14 \\
\hline Keine Expertenintervention (E) & 9 & 15 & 10 \\
\hline Prozessstabilität $(\mathrm{E})$ & 4 & 4 & 6 \\
\hline Prozesskontext $(\mathrm{E})$ & 3 & 5 & 5 \\
\hline Überschaubares Regelwerk (E) & 2 & 5 & 4 \\
\hline Transparente Prozesspfade (E) & 3 & 3 & 3 \\
\hline Neue Regeln (E) & 3 & 3 & 3 \\
\hline Vorhandene Prozessdokumentation (E) & 8 & 14 & 1 \\
\hline Outgesourcte Prozesse (E) & 1 & 1 & 1 \\
\hline \multicolumn{4}{|l|}{ Quantitative Prozesseigenschaften } \\
\hline Kosten-Nutzen-Vergleich (E) & 8 & 20 & 27 \\
\hline Anzahl Prozessinstanzen (E) & 10 & 26 & 25 \\
\hline Kompensation von Mitarbeiterkapazitäten (B) & 9 & 16 & 19 \\
\hline Reduktion von Durchlaufzeiten (B) & 8 & 14 & 11 \\
\hline Wenige Prozessschritte (E) & 5 & 8 & 9 \\
\hline Dynamische Prozesslast (E) & 5 & 6 & 6 \\
\hline Wenige Ausnahmefälle (E) & 4 & 5 & 4 \\
\hline Kurze Bearbeitungszeit (E) & 4 & 7 & 3 \\
\hline \multicolumn{4}{|l|}{ Risiko und Compliance } \\
\hline Compliance Konformität (E) & 10 & 18 & 17 \\
\hline Sicherstellung von Datenschutz (E) & 7 & 12 & 16 \\
\hline Vermeidung von menschlichem Irrtum (B) & 8 & 12 & 10 \\
\hline Vermeidung von Kopfmonopolen (B) & 8 & 11 & 10 \\
\hline Mehr Transparenz (B) & 2 & 5 & 4 \\
\hline
\end{tabular}

in negative (-1), neutrale (0), positive (1) oder besonders positive (2) Aussagen gruppiert.

Die Summe der Relevanzwerte einer Kategorie dient als Kennzahl für die Sortierung der Selektionskriterien. Zudem bildet der Mittelwert der Relevanz einen Treshold für überdurchschnittlich relevante Kriterien. Eine weitere Kennzahl stellt 
Tab. 2 (Fortsetzung)

\begin{tabular}{|c|c|c|c|}
\hline Dimension und Selektionskriterium & $\begin{array}{l}\text { Interview- } \\
\text { Frequenz }\end{array}$ & $\begin{array}{l}\text { Aussagen- } \\
\text { Frequenz }\end{array}$ & Relevanz \\
\hline \multicolumn{4}{|l|}{ Informationssysteme } \\
\hline Sinnvolle Brückenlösung (E) & 11 & 32 & 24 \\
\hline Systeminteraktion (E) & 5 & 14 & 14 \\
\hline Anzahl involvierter Systeme (E) & 11 & 27 & 10 \\
\hline Seltene Systemanpassungen (E) & 4 & 7 & 6 \\
\hline Systemberechtigungen (E) & 4 & 4 & 4 \\
\hline Keine neuen Systeme (E) & 5 & 5 & 3 \\
\hline \multicolumn{4}{|l|}{ Aufgabengebiete } \\
\hline Repetitive Aufgaben (E) & 9 & 15 & 15 \\
\hline Operative Prozesse (E) & 6 & 10 & 11 \\
\hline Aufwändige Vorarbeiten (E) & 6 & 10 & 10 \\
\hline Migration (E) & 3 & 3 & 3 \\
\hline Kein direkter Kundenkontakt (E) & 7 & 13 & 0 \\
\hline \multicolumn{4}{|l|}{ Entwicklung } \\
\hline Schnelle Automatisierung (B) & 9 & 14 & 10 \\
\hline Einfache Entwicklung (E) & 6 & 12 & 8 \\
\hline Wiederverwendbare Artefakte (B) & 5 & 12 & 5 \\
\hline Kapazitäten für Wartung und Pflege der Bots (E) & 3 & 5 & 3 \\
\hline Keine Entwicklung auf produktiver Umgebung (E) & 2 & 2 & 3 \\
\hline Verfügbare RPA-Entwickler (E) & 2 & 2 & 2 \\
\hline Produktionsnahe Systemumgebungen & 1 & 1 & 1 \\
\hline
\end{tabular}

(B) Beitrag zur Zielerreichung, (E) Eignung des Prozesses für RPA

die Interviewfrequenz dar, welche die Anzahl der Experten wiederspiegelt, die sich zu einer bestimmten Kategorie geäußert haben (Vogelsang et al. 2013). Die Grundannahme ist dabei, dass Kategorien mit einer hohen Interviewfrequenz eine höhere Bedeutung haben (Vogelsang et al. 2013). Zudem wird die Gesamtheit aller kodierten Aussagen einer Kategorie durch die Frequenz ausgedrückt.

\section{Ergebnisse}

\subsection{Untersuchungsszenario und Überblick der Selektionskriterien}

Die Untersuchung wurde bei einem mittelständischen deutschen Versicherungskonzern, der eine dreistellige Anzahl von Geschäftsprozessen ausführt, und einem für den Versicherer tätigen Beratungshaus durchgeführt. Der Konzern befindet sich zum Zeitpunkt der Untersuchung in einer Phase der Rationalisierung, die unter anderem durch den Einsatz von IT begleitet werden soll. In die Untersuchung sind sowohl einzelne Tochtergesellschaften (TG) als auch die Dachgesellschaft (DG) des Konzerns sowie ein Beratungshaus als externer Dienstleister (ED) involviert. In Summe wurden elf RPA-Experten interviewt, wobei deren Fachexpertise mindestens einer 
der RPA-Rollen nach Smeets et al. (2019) entsprechen muss. Tab. 1 beinhaltet die demografischen Daten aller Interviewteilnehmer.

Aus den Transkripten der elf Experteninterviews ergeben sich 576 Aussagen, die in insgesamt 51 induktiv gebildeten Kategorien bzw. Selektionskriterien zusammengefasst werden. Aufbauend auf den Selektionskriterien werden die Dimensionen Prozess-Stakeholder, Qualitative Prozesseigenschaften, Quantitative Prozesseigenschaften, Risiko und Compliance, Informationssysteme, Aufgabengebiete und Entwicklung gebildet und die einzelnen Kriterien diesen Dimensionen zugeordnet. Die Interview-Frequenz, die Häufigkeit der Zuordnungen von codierten Textstellen (Aussagen-Frequenz) sowie die Relevanz-Werte sind in Tab. 2 abgebildet. Der Mittelwert der Relevanzwerte liegt bei zehn. Ausgegraute Selektionskriterien liegen unterhalb dieses Grenzwertes und werden in diesem Beitrag nicht weiter erläutert. Mittels der Experten ID aus Tab. 2 wird auf Aussagen der Interviewteilnehmer verwiesen.

\subsection{Prozess-Stakeholder}

Die Dimension Prozess-Stakeholder bildet Kriterien ab, die einen Zusammenhang mit Beteiligten, die Einfluss auf die Prozessautomatisierung haben, aufweisen. Mit einem Relevanzwert von 33 ist die Arbeitnehmerüberlastung das wichtigste Selektionskriterium für geeignete RPA-Prozesse. Primär sollen Prozesse, in denen Überlastungen der Arbeitnehmer und Rückstände auftreten, automatisiert werden. Nach Ansicht von Interviewteilnehmer (Intln) C ist es wichtig, ,Freiräume zu schaffen für Tätigkeiten, die wertschöpfend sind, also [...] sich auf das uhreigene Geschäft fokussieren“ (C). Die Verfügbarkeit von Fachkräften steht oft mit dem Druck und den eingeplanten Kapazitäten der Linientätigkeit eines Arbeitnehmers in Konflikt. Um regelmäßig Feedback zur RPA-Entwicklung geben zu können, ist ,,vor allen Dingen mit [den] Führungskräften abzuklären, dass diese fachlichen Mitarbeiter auch freigestellt werden“"(B).

Bei der Auswahl eines Prozesses soll zudem der Mehrwert für den Kunden berücksichtigt werden (Steigerung der Kundenzufriedenheit). Dieser kann durch direkte Auswirkungen, wie z. B. schnellere Verarbeitung und geringere Wartezeiten (A, C, D) oder durch indirekte Auswirkungen, wie etwa die bereits erläuterte Entlastung der Mitarbeiter (B, F) erreicht werden. Sachbearbeiter erhalten so mehr Zeit für ,das Bearbeiten der Fälle, die wirklich eine Kundenzufriedenheit generieren" (D).

Der Vorschlag eines Fachbereichs für eine Prozessoptimierung mit RPA ist ein weiteres deutliches Selektionskriterium, das von sechs Interviewteilnehmern erwähnt wurde. Es ist ,extrem wichtig [...], dieses persönliche Empfinden von Mitarbeitern mit in die Prozessauswahl einzubeziehen“ (F) und sich durch ,direkte Gespräche mit den operativen Einheiten“ (E) der Frage zu widmen: „Welcher Prozess stört den Fachbereich und die Mitarbeiterinnen und Mitarbeiter am meisten?“(F). Analog wird auch die Akzeptanz der Fachkräfte als relevantes Kriterium betrachtet. Intln. F ist der Auffassung, dass ,,...] diejenigen, die mit dem Roboter zusammenarbeiten müssen, als Hauptfaktor [betrachtet werden sollen], weil eine Roboterverarbeitung auch immer mit ihrer Akzeptanz steht oder fällt"“. Dies könne einerseits daran lie- 
gen, dass Mitarbeiter Ängste um ihren Arbeitsplatz entwickeln (A, F, J) und sich andererseits ,der Arbeitsprozess des Mitarbeiters verändert“(A).

\subsection{Qualitative Prozesseigenschaften}

Unter qualitativen Prozesseigenschaften werden die Kriterien gruppiert, die Eigenschaften eines geeigneten Prozesses, des Prozessumfeldes oder des Prozessmanagements verkörpern, jedoch nicht quantitativ im Sinne von Prozess-Kennzahlen messbar sind. Das wichtigste Kriterium in dieser Dimension ist mit einem Relevanzwert von 26 die regelbasierte Prozessteuerung. Ein RPA Use Case soll eine eindeutige und regelbasierte Prozesssteuerung besitzen (A, B, C, D, G, H, I, J, K), die auf einen RPA-Bot übertragen werden kann. Dieser erhält dann die Fähigkeit, Entscheidungen zu treffen, die ,scharf formulierbar und in dem Sinne auch überprüfbar [sind]“ (J). Zudem sollen die Prozesse einen niedrigen Komplexitätsgrad besitzen. ,Je simpler der Prozess ist, desto eher können Mitarbeiter vielleicht darauf verzichten“ (F). Zwei Experten äußern auch ihre Bedenken, denn, ,einfache Tätigkeiten [gelten] auch als sogenannte Entlastungstätigkeiten“ (H) für die Mitarbeiter. Ausschließlich komplexe Tätigkeiten könnten irgendwann zu Überforderung führen (E).

RPA-Systeme benötigen Zugriff auf strukturierte Daten (B, C, H, G). Intl. I fragt etwa: „Wie komme ich an die erforderlichen Daten? Liegen sie mir strukturiert vor?“. Ohne den Zugriff auf strukturierte Daten, ist die Automatisierung nicht oder nur mit größerem Aufwand möglich (A, B, G, J). „Ich kann ein PDF als Bild oder als strukturierte XML-Struktur vorliegen haben. Das eine kann ich mit einem Roboter super technisch auslesen, für das andere brauche ich OCR, Optical Character Recognition“, erläutert F. Mit einem Relevanzwert von 14 ist die vorangegangene Prozessoptimierung ein weiteres relevantes Selektionskriterium. Dazu beziehen sich die Äußerungen entweder auf bereits erfolgte Optimierungen (B) oder während der RPA-Entwicklung erkannte Optimierungspotentiale (A). „Bestenfalls ist der Prozess vorher schon optimiert [...] und irgendwie [...] standardisiert worden [...]. Dann macht es ja wirklich erst Sinn den zu automatisieren. Das ist sehr wichtig“, betont B.

Für die Bearbeitung des Prozesses sollte keine Expertenintervention benötigt werden (A, B, D, I). In einigen internen Praxisfällen ist der einzige manuelle Schritt die Weiterleitung an den RPA-Bot durch den Mitarbeiter, ,weil der einen besseren Blick darauf hat, was der Bot kann und was [...] nicht. Und dann macht der Bot eigentlich von vorne bis hinten im Optimalfall alles [...], sodass der Mitarbeiter den auch nie wiedersieht" (A).

\subsection{Quantitative Prozesseigenschaften}

Die quantitativen Prozesseigenschaften umfassen Eigenschaften eines Prozesses, die eindeutig messbar in Form von Prozess-Kennzahlen sind. Mit einem Relevanzwert von 27 ist der Kosten-Nutzen-Vergleich das wichtigste Selektionskriterium dieser Dimension. Ein RPA Use Case sollte rentabel und effizienzsteigernd sein (A, C, D, G, H, J). Den Nutzen definieren die Experten häufig anhand der erreichbaren Zeitersparnis des agierenden Bots gegenüber einer manuellen Bearbeitung (A, C, D, G, J). Ein Experte betont, dass man die möglichen Use Cases ,im 
Gesamtkontext betrachten“ und auf Grund begrenzter Kapazitäten den Aufwand und Nutzen zwischen den Use Cases abwägen muss (C). Auch während der Automatisierung muss die Bewertung von Kosten und Nutzen nachgehalten werden $(\mathrm{H})$. Hierzu berichtet ein Experte: „Früher hat man alles automatisiert, was irgendwie ging und [...] dann [...] festgestellt, das war jetzt so teuer, diesen Geschäftsvorfall zu automatisieren, da hätten wir eigentlich auch problemlos einfach zwei Mitarbeiter einstellen können“ (D). Fast allen Experten erwähnen zudem das Kriterium Anzahl der Prozessinstanzen. Der Prozess sollte ,in einer ausreichend hohen Stückzahl [...] durchgeführt“(D) ,werden [...], um für eine Automatisierung in Frage zu kommen“. Ein simpler „Prozess, der aber auch sehr häufig auftritt, [kann] sehr schnell eine spürbare Entlastung beim Fachbereich erzeugen“(F). C ist der Auffassung, dass „30, 40 Fälle pro Tag [...], vielleicht 100 in der Woche“ auftreten sollten, um einen Nutzen durch RPA zu erhalten.

Die Kompensation von Mitarbeiterkapazitäten, die mit Hilfe der Bots erreicht wird, spielt nach Aussagen der Experten vor allem in der betreffenden Versicherung eine zentrale Rolle, da zahlreiche Mitarbeiter in der Vergangenheit das Unternehmen verlassen haben $(\mathrm{C}, \mathrm{D}, \mathrm{G}, \mathrm{H})$. Intln $\mathrm{D}$ formuliert dies wie folgt: „Also stellt sich für uns eigentlich gar nicht mehr die Frage: Machen wir das, um jetzt irgendwie Kosten zu sparen? Darum machen wir es nicht. Oder machen wir es eben, um das Arbeitsvolumen noch hinzukriegen?“ (D). Demnach konzentriert sich auch die Priorisierung auf Use Cases mit einer hohen MAK Kompensation (H).

Die notwendige Reduktion von Durchlaufzeiten ist ebenfalls ein relevantes Kriterium. Nach Auffassung von Intln. C hat RPA das Potential, „Durchlaufzeiten der Prozesse zu verkürzen“. Ein Roboter ist in der Lage, die Benutzeroberflächen schneller als ein Mensch zu bedienen (A, B, C, F, K). Nur eine längere systembedingte Ladezeit kann den Bot verlangsamen $(F)$. Unabhängig davon ist ein Roboter in der Lage, ,direkt wieder [zu starten], während ein Mitarbeiter natürlich [eine] ganz andere Reaktionszeit [...] an den Tag legt" (F).

\subsection{Risiko und Compliance}

Die Auswahlkriterien die zur Einhaltung von Gesetzen und Richtlinien führen, werden in der Dimension Risiko und Compliance gruppiert, wobei die Compliance Konformität das wichtigste Kriterium ist. Demnach werden Prozesse aussortiert, die aus Compliance-Gründen nicht umgesetzt werden können (A). Beispielsweise „dürfen [...] keine Entscheidungen zu Lasten des Kunden automatisiert getroffen werden“ (A). Zur Verhinderung von Betrugspotential berichten zwei Experten von einem zu unsicheren Use Case, welcher Zahlungsanweisungen von außen per Mail an einen Roboter senden sollte (A, K): „Wenn ich irgendwo aus Postfächern [...] Mails extrahiere und daraus Zahlungen ableite, ist das sehr gefährlich. Da könnte [...] ich mir ja einfach eine Mail reinschicken und dann kriege ich $100.000 €$ “" $(\mathrm{K})$. Sicherstellung von Datenschutz ist relevant für die Auswahl von Prozessen. Anhand eines Datenschutztemplates wird bewertet, ob es sich ,um datenschutzrechtlich bedenkliche Grundkonzepte [handelt]“ (F).

Die Vermeidung von menschlichem Irrtum umfasst ein weiteres relevantes Selektionskriterium, denn RPA ist in der Lage, in bestimmten Bereichen die Fehlerfreiheit 
und somit auch die Qualität der Prozessbearbeitung zu erhöhen (C, D, I). Hierzu beschreibt Interviewteilnehmer F: „Der Fakt einer Automatisierung ist nun mal, dass ein Roboter sein Regelwerk simpel abarbeitet und das Ganze fehlerfrei macht". Demnach ,lohnt es sich [...] gerade da [...], wo viele Fehler passieren können“ (D).

Die Vermeidung von Kopfmonopolen beim Einsatz von RPA (A, B, C, D, F, H) wird auch häufig erwähnt. Intln. A berichtet, dass mit Hilfe von Makros Prozesse durch die Fachbereiche bereits automatisiert werden. Gleichzeitig legen einige Mitarbeiter der Fachbereiche ,eine extrem hohe Eigenmotivation an den Tag“ (F) und erlernen Skriptsprachen, um sie im beruflichen Kontext einzusetzen. „Das Problem ist, dass es immer Kopfmonopole sind“ (F). Sobald der Mitarbeiter nicht mehr verfügbar ist, wird die Lösung, ,bald nicht mehr funktionieren, weil neue Software Versionen ausgerollt werden. Dann kann niemand dieses Tool anpassen“ (F). Um diese ,Schatten-IT“ (A, B) zu verdrängen, stellt RPA eine ,nachhaltige Lösung“ (A) dar.

\subsection{Informationssysteme}

Die Dimension Informationssysteme umfasst sämtliche Selektionskriterien, die sich auf die existierenden prozessrelevanten Systeme, ihre manuelle Bedienung bzw. die Schnittstellen und ihre Auswirkungen auf die Automatisierung beziehen. Ein von allen Experten angesprochenes, relevantes Kriterium ist die Wahl einer sinnvollen Brückenlösung, da eine saubere alternative Lösung nicht möglich oder zu aufwändig ist bzw. temporär kompensiert werden soll. Denn ,,nicht alles kann immer durch ein richtiges IT-Projekt gestemmt werden [...]. Dafür sind meistens nicht die Ressourcen gegeben“ (A). Intln. K sagt dazu: „Natürlich wäre es geschickter, die Bestandssysteme zu verheiraten [...]. Da aber momentan ja eben diese Host-Ablösung geplant ist, brauchen wir im Host nichts mehr dazuprogrammieren“.

Eng mit einer sinnvollen Brückenlösung verbunden ist die Fähigkeit zur Systeminteraktion der existierenden Oberflächen. „Gerade in einer heterogenen Architekturlandschaft, wie es in der Versicherungsbranche üblich ist, [existieren] Systeme, die der Roboter-Automatisierung günstiger [...] und [...] nicht so günstig gegenüberstehen“ (F). Dies zeigt zum Beispiel der Vergleich zwischen ,Softwareframeworks der 70er-Jahre“ (F) und einer ,Web Anwendung, die sich über einen modernen Browser ganz leicht automatisieren lässt" (D).

Ein weiteres relevantes und gleichzeitig umstrittenes Kriterium ist die Anzahl der Systeme, die ein für RPA geeigneter Prozess beinhalten sollte. Einerseits empfiehlt die Mehrheit der Experten, dass gerade ein Prozess, der ,,idealerweise von Medienbrüchen gezeichnet ist, also wo [der Sachbearbeiter] verschiedene IT-Systeme in irgendeiner Form bedienen muss" (D) für die Automatisierung mit RPA geeignet ist. Der Nutzen der Maßnahme wird dabei ,,potenziell immer größer, je mehr Systeme beteiligt sind“ (D), denn eine existierende Automatisierung über Makros bietet keine Möglichkeit, systemübergreifend Prozesse zu verarbeiten (D, H). Andererseits spricht gegen eine hohe Anzahl involvierter Systeme, dass die Komplexität des ,gesamten Entwicklungsprozesses, von der Businessanalyse über die [...] Instandhaltung und Wartung“ steigt (C). 


\subsection{Aufgabengebiete und Entwicklung}

Die Dimension Aufgabengebiete umfasst Kriterien, die konkrete Einsatzgebiete, Aufgaben und Bereiche kennzeichnen, die für eine RPA-Bearbeitung besonders gut geeignet sind. Mit einem Relevanzwert von 15 sind vor allem repetitive Aufgaben relevant für RPA. Die Automatisierung dieser Aufgaben erzeugt einen entlastenden Effekt für die Mitarbeiter, da Aufgaben ,die einen gewissen monotonen Charakter haben“ (K) von den Mitarbeitern als ,,ermüdend und als fast schon degradierend“ (J) empfunden werden können. Hierzu liefert einer der Experten ein Beispiel, bei dem ,jeden Tag um 12 Uhr ein Stahlpreis ermittelt werden [muss], der dann wiederum ins SAP übertragen wird“ (K). Des Weiteren sollten vor allem operative Prozesse für die Automatisierung ausgewählt werden (C, D, F, G, H). „Ich denke, dass definitiv unsere operativen Bereiche [...] die meisten Potentiale darstellen, was primär auch einfach an den hohen [...] Vorgangszahlen und einem hohen Grad von Standardisierung, der jetzt schon vorherrscht, liegt" (F).

Das relevante Selektionskriterium Aufwändige Vorarbeiten umfasst Arbeitsschritte, die von einem Mitarbeiter erledigt werden müssen, bevor die eigentliche Arbeit begonnen werden kann. Gerade ,das saubere Routen von Geschäftsvorfällen [...] zu den richtigen Mitarbeitern oder zu den richtigen Teams“ (B) ist ein Beispiel für aufwändige Arbeiten, bevor mit der eigentlichen Bearbeitung begonnen werden kann. Außerdem wird beschrieben, dass Bots erfolgreich zur Beschaffung und Zusammenfassung relevanter Daten verschiedenster Quellen eingesetzt werden können, sodass der Bot den Empfänger ,nicht nur informationell unterstützt und ihm den Rechercheaufwand spart, sondern [...] ihm wirklich Teilprozesse komplett wegnimmt“"(I).

Das Potenzial, schnelle Automatisierungen durchführen zu können und dementsprechend dringende Use Cases zu bevorzugen, verkörpert das einzig relevante Kriterium der Dimension Entwicklung. Diesbezüglich berichtet ein Experte, dass der Anspruch des (heutigen) Managements ist, dass entsprechende Maßnahmen kurzfristig, ,,am besten noch dieses Quartal“ Ergebnisse erzielen müssen (A). Im Vergleich zu klassischeren Methoden, die ,sehr viel Zeit in Anspruch nehmen, bis [...] überhaupt die nötigen Entwickler“ bereitgestellt werden, sind die „Entwicklungszyklen [...] ausgesprochen kurz“ (J).

\section{Diskussion und Ausblick}

Vergleicht man die Ergebnisse der Interviews mit den bekannten Kriterien der Literatur, so fällt auf, dass die Kriterien Regelbasierte Prozesssteuerung, Niedriger Komplexitätsgrad, Repetitive Aufgaben, Viele Prozessinstanzen, Kompensation von Mitarbeiterkapazitäten, Digital vorhandene strukturierte Daten, Optimierter Prozess und Sinnvolle Brückenlösung, sowohl in der Literatur als auch in den Interviews von Bedeutung für die Prozessselektion sind.

Das Kriterium Transparente Prozess-Istkosten wird in der Literatur oft angegeben (Beetz und Riedl 2019; Smeets et al. 2019; Sutherland 2013; Syed et al. 2020), jedoch von den Experten nicht explizit erwähnt. Des Weiteren wurden von Syed et al. (2020) die notwendige Akzeptanz der Kunden sowie die hohe Datenqualität bei 
den RPA-Eingabedaten als weitere Kriterien aufgeführt, was ebenfalls nicht durch die befragten Experten adressiert wird. Beetz und Riedl (2019) benennen außerdem die Fehlertoleranz eines Prozesses, welcher automatisiert bearbeitet werden soll, als Selektionskriterium.

Die Gegenüberstellung der Kriterien ergibt acht relevante Kriterien, die bislang in der Literatur nicht oder unzureichend diskutiert werden. Hierzu zählen die Kriterien Arbeitnehmerüberlastung, Compliance Konformität, Sichergestellter Datenschutz, Steigerung der Kundenzufriedenheit, Systeminteraktion, Operative Prozesse, Vermeidung von Kopfmonopolen und Aufwändige Vorarbeiten. Sie bilden die Grundlage für weitere Untersuchungen.

Trotz methodisch fundierter Vorgehensweise (Vogelsang et al. 2013), ist die Aussagekraft der Ergebnisse begrenzt. So wurde lediglich ein Konzernverbund und ein Beratungshaus in den Interviews berücksichtigt. Auch die starke Ausprägung männlicher Interviewteilnehmer kann die Ergebnisse beeinflussen. Wie in jeder qualitativempirischen Arbeit sind die Codierungsergebnisse zu einem Teil auch von der subjektiven Wahrnehmung des Codierers abhängig. Aufbauende Arbeiten sollten daher die erzielten Ergebnisse durch weitere qualitative Untersuchungen oder durch eine Umfrage in anderen Organisationen und Branchen validieren bzw. weitere Kriterien identifizieren. Zudem sind die Unterschiede in der Relevanz der einzelnen Kriterien in verschiedenen Wirtschaftszweigen und deren Einfluss auf den Projekterfolg eine weitergehende Betrachtung wert.

Funding Open Access funding enabled and organized by Projekt DEAL.

Open Access Dieser Artikel wird unter der Creative Commons Namensnennung 4.0 International Lizenz veröffentlicht, welche die Nutzung, Vervielfältigung, Bearbeitung, Verbreitung und Wiedergabe in jeglichem Medium und Format erlaubt, sofern Sie den/die ursprünglichen Autor(en) und die Quelle ordnungsgemäß nennen, einen Link zur Creative Commons Lizenz beifügen und angeben, ob Änderungen vorgenommen wurden.

Die in diesem Artikel enthaltenen Bilder und sonstiges Drittmaterial unterliegen ebenfalls der genannten Creative Commons Lizenz, sofern sich aus der Abbildungslegende nichts anderes ergibt. Sofern das betreffende Material nicht unter der genannten Creative Commons Lizenz steht und die betreffende Handlung nicht nach gesetzlichen Vorschriften erlaubt ist, ist für die oben aufgeführten Weiterverwendungen des Materials die Einwilligung des jeweiligen Rechteinhabers einzuholen.

Weitere Details zur Lizenz entnehmen Sie bitte der Lizenzinformation auf http://creativecommons.org/ licenses/by/4.0/deed.de.

\section{Literatur}

van der Aalst WMP, Bichler M, Heinzl A (2018) Robotic process automation. Bus Inf Syst Eng 60:269-272. https://doi.org/10.1007/s12599-018-0542-4

Aguirre S, Rodriguez A (2017) Automation of a business process using robotic process automation (RPA): a case study. In: Figueroa-García et al (Hrsg) 4th Workshop on Engineering Applications, WEA. Springer, Berlin Heidelberg, S 65-71

Beetz R, Riedl Y (2019) Robotic process automation: developing a multi-criteria evaluation model for the selection of automatable business processes. 25th Americas Conference on Information Systems (AMCIS).

Fung HP (2014) Criteria, use cases and effects of information technology process automation (ITPA). Adv Robot Autom. https://doi.org/10.4172/2168-9695.1000124 
Lacity M, Willcocks L (2016) Robotic process automation at telefonica O2. MIS Q Executiv 15(1):21-35 Mayring P (2010) Qualitative Inhaltsanalyse. In: Mey G, Mruck K (Hrsg) Handbuch Qualitative Forschung in der Psychologie. VS, Wiesbaden, S 601-613

Smeets M, Erhard R, Kaußler T (2019) Robotic Process Automation (RPA) in der Finanzwirtschaft; Technologie - Implementierung - Erfolgsfaktoren für Entscheider und Anwender. Springer, Berlin Heidelberg

Sutherland C (2013) Framing a constitution for robotistan; racing with the machine of robotic automation. HfS Research Ltd, https://www.hfsresearch.com/research/framing-constitution-robotistan/. Zugegriffen: 12.10 .2020 .

Syed R, Suriadi S, Adams M, Bandara W, Leemans SJJ, Ouyang C, ter Hofstede AHM, van de Weerd I, Wynn MT, Reijers HA (2020) Robotic process automation: contemporary themes and challenges. Comput Ind 115:103162. https://doi.org/10.1016/j.compind.2019.103162

Vogelsang K, Steinhueser M, Hoppe U (2013) A qualitative approach to examine technology acceptance. 34th International Conference on Information Systems (ICIS 2013).

Willcocks L, Lacity M, Craig A (2015) The IT function and robotic process automation. https://www.uio. no/studier/emner/matnat/ifi/ITLED4021/h18/dokumentasjon/the-it-function-and-robotic-processautomation-willcocks-2015.pdf. Zugegriffen: 24. Juni 2020 